\title{
Spectroscopy of the Dirac oscillator perturbed by a surface delta potential
}

\author{
J. Munárriz and F Domínguez-Adame \\ GISC. Departamento de Física de Materiales, Universidad Complutense, E-28040 \\ Madrid, Spain \\ R. P. A. Lima \\ Instituto de Física, Universidade Federal de Alagoas, Maceió, AL 57072-970, Brazil
}

\begin{abstract}
We study theoretically the level shift of the Dirac oscillator perturbed by any sharply peaked potential approaching a surface delta potential. A Green function method is used to obtain closed expressions for all partial waves and parities.

Keywords: Dirac oscillator, singular potentials, relativistic wave equations PACS: 03.65.pm, 03.65.-w, 03.65.Ge,
\end{abstract}

Physics Letters A 376, 3475 (2012)

According to their behavior under Lorentz transformations, the interaction potentials for the Dirac equation are either vector, scalar or pseudoscalar. Here the term vector means the time component of a Lorentz vector, while a scalar potential is equivalent to a dependence of the mass upon position. Relativistic wave equations with vector and scalar linearly rising potentials have been widely used to investigate the confinement of particles in nuclear and hadron physics. When the linear potential is vectorlike, there exist no bound states and only tunneling solutions arise $[1,2]$. Therefore, vector linear potentials cannot confine particles, being another fine example of the Klein tunneling [3]. On the contrary, scalar linear potentials can bind relativistic particles and give rise to confinement since the mass of the particle increases 
without bound when the separation from the center gets larger $[2,4,5]$. Similar conclusions can be drawn for vector and scalar parabolic potentials [6].

In spite of the fact that vector linear and quadratic potentials cannot bind particles, it is possible to extend the quantum harmonic oscillator to the relativistic domain [6]. In this context, Moshinsky and Szczepaniak introduced a new type of interaction in an attempt to describe a relativistic oscillator by means of a Dirac equation linear in both momenta and coordinates [7]. The authors gave the name of Dirac oscillator to this model, whose nonrelativistic limit leads to a standard harmonic oscillator equation with spin-orbit term. The complete energy spectrum and the electromagnetic potential associated to the Dirac oscillator were found by Benítez et al. [8]. The spectrum presents degeneracies which are explained by a symmetry Lie algebra [9]. Regardless the intensity of the coupling, this interaction leaves the vacuum unchanged and the Klein tunneling is avoided [8, 10]. Therefore, in contrast to the vector linear potential, the resulting states are truly bound and the Dirac oscillator is a good candidate to explain the observed confinement of quarks [11]. This interaction has also been considered in the two-body Dirac equation, presenting interesting features which are not shared with the onebody oscillator [12]. Recently, a photonic realization of the Dirac oscillator based on light propagation in engineered fiber Bragg gratings has been discussed by Longhi [13]. Therefore, the interest of the Dirac oscillator is rather general and well beyond high energy physics.

The aim of this paper is to study the spectroscopy of the Dirac oscillator in a $(3+1)$-dimensional space perturbed by a surface delta potential, solving the associated Lippmann-Schwinger equation. Therefore, we present a generalization of the results obtained in Ref. [14], in the sense that we do not restrict ourselves to $(1+1)$-dimensional space. In addition, in Ref. [14] the perturbation was described by a nonlocal separable potential. The $\delta$-function limit of a nonlocal potential in the Dirac equation is mathematically well defined [15] and the corresponding Lippmann-Schwinger equation is valid even in this limit. However, as discussed in Ref. [16], there exist some ambiguities in defining the surface delta potential (local) that require a careful analysis of the Lippmann-Schwinger equation, as we show below.

We arrive at the Dirac oscillator equation by the non-minimal substitution $\boldsymbol{p} \rightarrow \boldsymbol{p}-i m \omega \beta \boldsymbol{r}$, where $m$ is the mass of the particle, $\omega$ is the oscillator frequency and $\beta$ is the usual Dirac matrix defined below. From the above considerations, the Dirac Hamiltonian may be written in the standard nota- 
tion as (in units with $\hbar=1$ and $c=1$ )

$$
\mathcal{H}=\boldsymbol{\alpha} \cdot(\boldsymbol{p}-i m \omega \beta \boldsymbol{r})+\beta m+V_{R}(r) \equiv \mathcal{H}_{0}+V_{R}(r) .
$$

We choose the representation

$$
\boldsymbol{\alpha}=\left(\begin{array}{cc}
0 & \boldsymbol{\sigma} \\
\boldsymbol{\sigma} & 0
\end{array}\right), \quad \boldsymbol{\beta}=\left(\begin{array}{cc}
I_{2} & 0 \\
0 & -I_{2}
\end{array}\right),
$$

where $\boldsymbol{\sigma}=\left(\sigma_{x}, \sigma_{y}, \sigma_{z}\right)$ contains the Pauli matrices and $I_{2}$ is the $2 \times 2$ unity matrix.

We are interested in the energy spectrum of the Dirac oscillator, whose Hamiltonian is given by $\mathcal{H}_{0}$ in (1), perturbed by a potential $V_{R}(r)$ approaching the $\delta$-shell limit with radius $R$. However, as mentioned above, the resulting equation is ambiguous if one takes the limit $V_{R}(r) \rightarrow \lambda \delta(r-R)$ from the outset. The origin of the ambiguity is the following. Since the Dirac equation is linear in momentum, the wavefunction itself must be discontinuous at $r=R$ to account for the singular term $V_{R}(r) \rightarrow \lambda \delta(r-R)$. However, the product of a discontinuous function and the $\delta$-function is mathematically ill defined. This ambiguity can be avoided by solving the corresponding Dirac equation for any arbitrary sharply peaked at $r=R$ function, $R$ being the radius of the shell, and then take the $\delta$-function limit with the constraint

$$
\int_{R-\Delta R}^{R+\Delta R} V_{R}(r) d r=\lambda, \quad \Delta R \rightarrow 0,
$$

where $\lambda$ is the dimensionless coupling constant.

For the moment, we only assume that the potential $V_{R}(r)$ is spherically symmetric. The eigenfunctions of definite parity and total angular momentum $\left(J^{2}, J_{z}\right)$ are written in the form

$$
\boldsymbol{\Psi}(\boldsymbol{r})=\frac{1}{r}\left(\begin{array}{c}
i f(r) \\
g(r) \boldsymbol{\sigma} \cdot \boldsymbol{r} / r
\end{array}\right) \boldsymbol{\Phi}_{j m_{j}}^{l},
$$

where $\boldsymbol{\Phi}_{j m_{j}}^{l}$ are the normalized two-components eigenfunctions of $J^{2}, J_{z}, L^{2}$ and $S^{2}[17]$. Using (1) and (3), the Dirac equation leads to

$$
\frac{d}{d r} \phi(r)=\left[\sigma_{z} \gamma(r)-\sigma_{x} m+i \sigma_{y}\left(E-V_{R}(r)\right)\right] \boldsymbol{\phi}(r)
$$

where the upper and lower components of the radial spinor $\phi(r)$ are $f(r)$ and $g(r)$, respectively. Here $\kappa=\mp(j+1 / 2)$ for $l=j \pm 1 / 2$ and we have 
defined $\gamma(r)=\kappa / r+m \omega r$ for brevity. Equation (4) is solved by a Newmann solution as follows [18]

$$
\phi(r)=\widehat{P} \exp \left\{\int_{r_{0}}^{r} d r^{\prime}\left[\sigma_{z} \gamma\left(r^{\prime}\right)-\sigma_{x} m+i \sigma_{y}\left(E-V_{R}\left(r^{\prime}\right)\right)\right]\right\} \boldsymbol{\phi}\left(r_{0}\right)
$$

where $\widehat{P}$ is the ordering operator. Setting $r=R+\Delta R$ and $r_{0}=R-\Delta R$, taking the limit $\Delta R \rightarrow 0$ and using the constraint (2) we finally obtain the following boundary condition

$$
\phi(R+\Delta R)=\exp \left(-i \lambda \sigma_{y}\right) \boldsymbol{\phi}(R-\Delta R),
$$

which becomes independent of how the $\delta$-function limit is taken and thus we avoid any ambiguity defining the relativistic surface $\delta$ potential.

Once the correct boundary condition at the singularity has been obtained, we proceed to find the energy spectrum of the perturbed Dirac oscillator. To this end, we write the Lippmann-Schwinger solution of the radial Dirac equation (4)

$$
\phi(r)=-\int_{R-\Delta R}^{R+\Delta R} \mathcal{G}\left(r, r^{\prime} ; E\right) V_{R}\left(r^{\prime}\right) \phi\left(r^{\prime}\right) d r^{\prime}
$$

where the Green function for the unperturbed problem is a $2 \times 2$ matrix satisfying the inhomogeneous differential equation

$$
\left[-i \sigma_{y} \frac{\partial}{\partial r}+\sigma_{x} \gamma(r)+\sigma_{z} m-E\right] \mathcal{G}\left(r, r^{\prime} ; E\right)=I_{2} \delta\left(r-r^{\prime}\right) .
$$

The Green function exhibits a jump discontinuity at the line $r=r^{\prime}$. The value of the jump can be obtained by integration of (7) in the vicinity of this line. The result is

$$
\mathcal{G}(r+\Delta R, r ; E)-\mathcal{G}(r-\Delta R, r ; E)=i \sigma_{y}
$$

The product $V_{R}(r) \phi(r)$ in the integral (6) is not well defined if one takes the limit $V_{R}(r) \rightarrow \lambda \delta(r-R)$, as we already discussed. Thus, we consider the same limiting procedure discussed previously and solve (6) for any arbitrary sharply peaked at $r=R$ function and then take the $\delta$-function limit. Using 
the radial Dirac equation (4) one finds that the integral equation (6) leads to (see Ref. [19] for details)

$$
\phi(r)=i \mathcal{G}(r, R ; E) \sigma_{y}[\phi(R+\Delta R)-\phi(R-\Delta R)] .
$$

Hence we have obtained a closed expression for the perturbed eigenfunctions. The energy levels can be obtained by setting $r=R+\Delta R$ in (9) and using the boundary condition (5)

$$
\operatorname{det}\left[I_{2}-i \mathcal{G}(R+\Delta R, R ; E) \sigma_{y}\left(e^{i \sigma_{y} \lambda}-I_{2}\right)\right]=0 \text {. }
$$

Therefore, the energy spectrum of the perturbed Dirac oscillator can be obtained provided the Green function for the unperturbed problem is known. The Green function of the Dirac oscillator can be cast in the form

$$
\mathcal{G}\left(r, r^{\prime} ; E\right)=\left(\begin{array}{cc}
G_{++}\left(r, r^{\prime} ; E\right) & G_{+-}\left(r, r^{\prime} ; E\right) \\
G_{-+}\left(r, r^{\prime} ; E\right) & G_{--}\left(r, r^{\prime} ; E\right)
\end{array}\right)
$$

and it was calculated in Ref. [20]. For the sake of brevity we only quote here the final expressions of the diagonal terms

$$
G_{ \pm \pm}=\frac{E \pm m}{m \omega} \frac{\Gamma\left(\nu_{ \pm}-\mu_{ \pm}+\frac{1}{2}\right)}{\Gamma\left(2 \nu_{ \pm}+1\right) \sqrt{r r^{\prime}}} M_{\mu_{ \pm}, \nu_{ \pm}}\left(m \omega r_{<}^{2}\right) W_{\mu_{ \pm}, \nu_{ \pm}}\left(m \omega r_{>}^{2}\right)
$$

where $M_{\mu, \nu}$ and $W_{\mu, \nu}$ are the Whittaker functions, $r_{<}=\min \left(r, r^{\prime}\right)$ and $r_{>}=$ $\max \left(r, r^{\prime}\right)$. The parameters $\mu_{ \pm}$and $\nu_{ \pm}$are defined as

$$
\mu_{ \pm}=\frac{1}{4}\left(\frac{E^{2}-m^{2}}{m \omega}-2 \kappa \pm 1\right), \quad \nu_{ \pm}=\frac{1}{2}\left|\kappa \pm \frac{1}{2}\right|
$$

The off-diagonal terms are calculated from the following expressions

$$
G_{ \pm \mp}=\frac{1}{E \mp m}\left[\mp \frac{\partial}{\partial r}+\frac{\kappa}{r}+m \omega r\right] G_{\mp \mp} .
$$

Real solutions of (10) with the Green function given by (11) yield the energy levels of the perturbed Dirac oscillator. Due to the boundary condition (5), the energy levels of the perturbed Dirac oscillator satisfy the property $E(m, \kappa, R, \lambda)=E(m, \kappa, R, \lambda+\ell \pi), \ell$ being an integer. Since the energy 
levels are $\pi$-periodic functions of the coupling constant, we can restrict ourselves to the range $-\pi / 2<\lambda \leq \pi / 2$ hereafter. Figure 1 shows these levels as a function of the coupling constant for the case $\omega=m$. The energy levels of the perturbed Dirac oscillator are shifted upwards on increasing the coupling constant from $-\pi / 2$ to $\pi / 2$. Notice that the levels of the perturbed Dirac oscillator cross those of the unperturbed oscillator only when $\lambda=n \pi, n$ being an integer. According to Eq. (5), in this case $\phi(R+\Delta R)=(-1)^{n} \phi(R-\Delta R)$ and the surface $\delta$ potential is actually transparent.
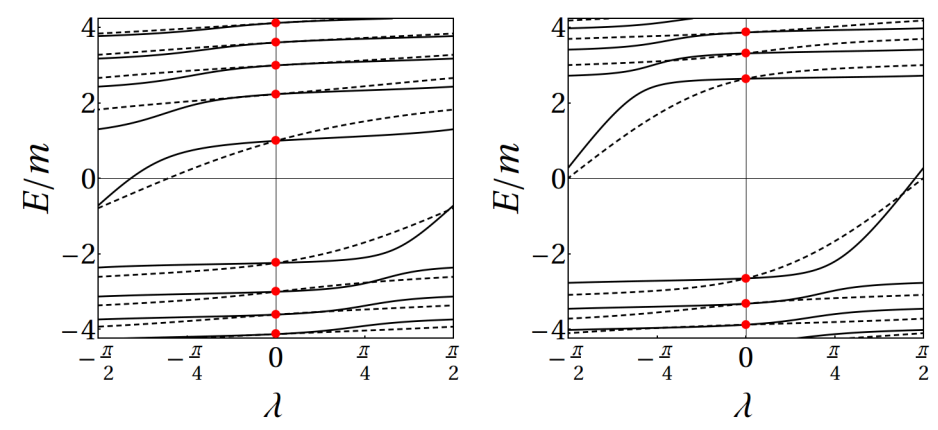

Figure 1: Energy levels of a Dirac oscillator perturbed by a surface delta potential as a function of the coupling constant $\lambda$ for $\omega=m$. Left and right panels correspond to $\kappa=-1$ and $\kappa=1$, respectively. Solid (dashed) lines correspond to the results for $R=0.3 / \mathrm{m}$ $(R=1 / m)$. The solutions of the unperturbed Dirac oscillator are marked with red points.

In the limit $\lambda \rightarrow 0$, the eigenvalues of the unperturbed system are recovered [21]. They correspond to the poles of $\mathcal{G}$, namely the set of values $E_{0}(m, \kappa)$ for which $\left(E_{0} \pm m\right) \Gamma\left(\nu_{ \pm}-\mu_{ \pm}+1 / 2\right) \rightarrow \infty$

$$
E_{0}^{2}(m, \kappa)-m^{2}=4 m \omega[n+\theta(\kappa)(2 \kappa+1)], \quad E_{0} \neq-m,
$$

where $\theta$ is the Heaviside step function and $n$ is a nonnegative integer.

Due to the confining properties of the Dirac oscillator, the spatial extent of the eigenstates increases with the absolute value of the energy, $|E|$. If this spatial extent is much smaller than the radius of the surface $\delta$ potential, the net effect of the perturbation on the eigenfunction is small, and therefore the energy does not change noticeably. This trend is clearly observed in Fig. 2, where the energy levels are plotted as a function of the radius $R$ for $\omega=m$, $\kappa=-1$ and $\lambda=\pi / 4$. When the dimensionless coupling constant is large, the energy levels display anticrossings, as seen in the figure. Moreover, each energy level approaches the same level of the unperturbed Dirac oscillator in the two limiting situations, $m R \ll 1$ and $m R \gg 1$. 


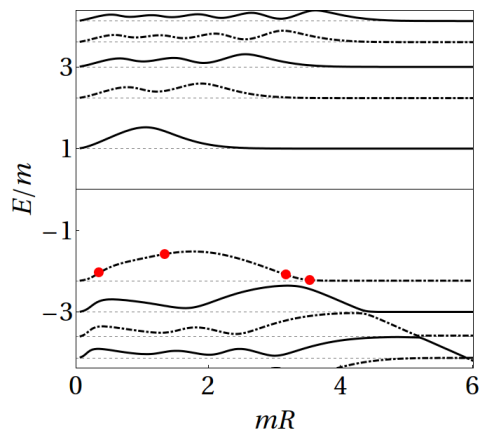

Figure 2: Shift of the energy levels as a function of the radius $R$ for $\omega=m, \kappa=-1$ and $\lambda=\pi / 4$. The levels of the unperturbed Dirac oscillator are plotted with dashed gray lines. The wave functions corresponding to the four red points are shown in Fig. 3 The wave functions at points marked $a-d$ are shown in figure 3 below.

Finally, the change of the probability density $|\phi(r)|^{2}$ due to the surface $\delta$ potential is shown in Fig. 3. The set of parameters chosen correspond to the four red circles in Fig. 2, i.e., all of them are taken from the same original unperturbed eigenstate. As $R$ is increased, the eigenstate transits between two loosely perturbed states - upper left and lower right panels. In between, states with probability density strongly peaked around $r=R$ are found.

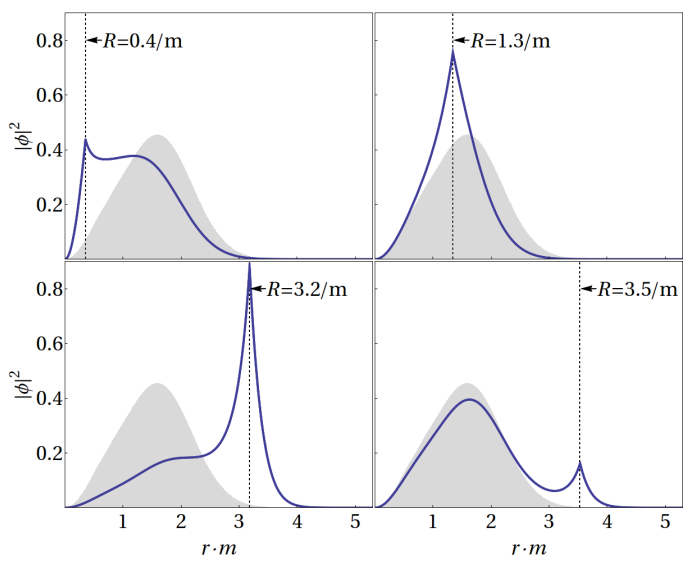

Figure 3: Plots of $|\phi(r)|^{2}$ as a function of the dimensionless radial coordinate $z=r \cdot m$ for the four different values of the delta-shell radius $R$ indicated in figure 2 by red circles, when $\omega=m, \kappa=-1$ and $\lambda=\pi / 4$. The corresponding unperturbed eigenstate is plotted using gray solid regions.

In summary, we have calculated the shift of the energy levels of the Dirac 
oscillator perturbed by a surface $\delta$ potential using a Green function technique. The method is valid for any sharply peaked potential approaching the $\delta$-function and consequently it is free of the ambiguities appearing in defining relativistic $\delta$-interactions [16]. Remarkably, the energy spectrum is a $\pi$-periodic function of the coupling constant $\lambda$, a situation not found in the $(1+1)$-dimensional Dirac oscillator perturbed by a nonlocal $\delta$ potential [14].

Work in Madrid was supported by MICINN (project MAT2010-17180). R. P. A. Lima would like to thank CAPES via project PPCP-Mercosul, CNPq, and FINEP (Brazilian Research Agencies) as well as FAPEAL (Alagoas State Research Agency) for partial financial support.

[1] M. L. Glasser and N. Shawagfeh, J. Math. Phys. 25 (1984) 2533.

[2] F. Domínguez-Adame and M. A. González, Europhys. Lett. 13 (1990) 193.

[3] H. Galic, Am. J. Phys. 56 (1988) 312.

[4] C. L. Critchfield, J. Math. Phys. 17 (1976) 261.

[5] B. Ram, J. Phys. A: Math. Gen. 20 (1987) 5023.

[6] F. M. Toyama and Y. Nogami, Phys. Rev. A 59 (1999) 1056.

[7] M. Moshinsky and A. Szczepaniak, J. Phys. A: Math. Gen. 22 (1989) L817.

[8] J. Benítez, R. P. Martínez y Romero, H. N. Núñez-Yepes and A. L Salas-Brito, Phys. Rev. Lett. 64 (1990) 1643.

[9] C. Quesne and M. Moshinsky, J. Phys. A: Math. Gen. 23 (1990) 2262.

[10] F. Domínguez-Adame, Phys. Lett. A 162 (1992) 18.

[11] M. Moreno and A. Zentella, J. Phys. A: Math. Gen. 22 (1989) L821.

[12] F. Domínguez-Adame and B. Méndez, Can. J. Phys. 69 (1991) 780.

[13] S. Longhi, Opt. Lett. 35 (2010) 1302.

[14] F. Domínguez-Adame, Europhys. Lett. 15 (1991) 569. 
[15] M. G. Calkin, D. Kiang and Y. Nogami, Phys. Rev. C 38 (1988) 1076.

[16] F. Domínguez-Adame, J. Phys. A: Math. Gen. 23 (1990) 1993.

[17] D. Bjorken and S. D. Drell, Relativistic Quantum Mechanics (Mc-Graw Hill, New York, 1964) p. 53.

[18] B. H. J. McKellar and G. J. Stephenson, Phys. Rev. A 36 (1987) 2566.

[19] B. Méndez and F. Domínguez-Adame, J. Phys. A: Math. Gen. 25 (1992) 2065.

[20] A. D. Alhaidari, Int. J. Theor. Phys. 43 (2004) 939.

[21] R.. Szmytkowski and M. Gruchowski, J. Phys. A: Math. Gen. 34 (2001) 4991. 